\title{
Metabolic Benefits of Methionine Restriction in Adult Mice Do Not Require Functional Methionine Sulfoxide Reductase a (MsrA)
}

Kevin M. Thyne

University of Texas Health San Antonio

Adam B.Salmon ( $\square$ Salmona@uthscsa.edu )

University of Texas Health San Antonio

\section{Research Article}

Keywords: glucose, redox, insulin, methionine, methionine sulfoxide, MsrA, methionine sulfoxide reductase $A$, methionine restriction, MetR

Posted Date: September 15th, 2021

DOI: https://doi.org/10.21203/rs.3.rs-859463/v1

License: (c) (i) This work is licensed under a Creative Commons Attribution 4.0 International License.

Read Full License

Version of Record: A version of this preprint was published at Scientific Reports on March 24th, 2022. See the published version at https://doi.org/10.1038/s41598-022-08978-4. 
Title:

Metabolic benefits of methionine restriction in adult mice do not require functional methionine sulfoxide reductase $\mathrm{A}$ (MsrA)

Authors:

Kevin M. Thyne ${ }^{1}$, Adam B. Salmon ${ }^{1,2,3,{ }^{*}}$

[1] Sam and Ann Barshop Institute for Longevity and Aging Studies, University of Texas Health San Antonio, San Antonio, Texas, 78229, USA

[2] Department of Molecular Medicine, University of Texas Health San Antonio, San Antonio, Texas. 78229, USA

[3] Geriatric Research Education and Clinical Center, Audie L. Murphy Hospital, South Texas Veterans Health Care System, San Antonio, Texas, 78229, USA

${ }^{*}$ Corresponding author

Kevin Thyne - Thyne@livemail.uthscsa.edu

*Dr. Adam Salmon - Salmona@uthscsa.edu

Keywords: glucose, redox, insulin, methionine, methionine sulfoxide, MsrA, methionine sulfoxide reductase $A$, methionine restriction, MetR 


\begin{abstract}
:
Methionine restriction (MR) extends lifespan and improves several markers of health in rodents. However, the proximate mechanisms of MR on these physiological benefits have not been fully elucidated. The essential amino acid methionine plays numerous biological roles and limiting its availability in the diet directly modulates methionine metabolism. There is growing evidence that redox regulation of methionine has regulatory control on some aspects of cellular function but interactions with MR remain largely unexplored. We tested the functional role of the ubiquitously expressed methionine repair enzyme methionine sulfoxide reductase $A$ (MsrA) on the metabolic benefits of MR in mice. MsrA catalytically reduces both free and protein-bound oxidized methionine, thus playing a key role in its redox state. We tested the extent to which MsrA is required for metabolic effects of MR in adult mice using mice lacking MsrA. As expected, MR in control mice reduced body weight, altered body composition, and improved glucose metabolism. Interestingly, lack of MsrA did not impair the metabolic effects of MR on these outcomes. Moreover, females had blunted MR responses regardless of MsrA status compared to males. Overall, our data suggests that MsrA is not required for the metabolic benefits of MR in adult mice.
\end{abstract}




\section{Introduction:}

Restriction of dietary intake of the amino acid methionine, even in the absence of restriction of calories, has been shown to consistently extend lifespan and improve metabolic health in rodents. Previous studies have shown that dietary methionine restriction (MR) in rodents can extend lifespan up to $42 \%$ compared to rodents fed diets replete with methionine ${ }^{[1-3]}$. Moreover, MR has strong effects on metabolic function and has been shown to improve glucose homeostasis ${ }^{[4-9]}$, decrease oxidative stress ${ }^{[2,10-15]}$, and promote adipose tissue browning $^{[16-22]}$. These findings are consistent with the idea that dietary intervention by restricting methionine improves health in addition to extending longevity. Understanding the molecular mechanisms responsible for the physiological effects of MR could then have significant impact as potential targets to improve human health throughout life.

While pro-longevity dietary interventions affect many molecular pathways consistent with the pillars of aging, it seems likely that the effects of MR may strongly affect methionine-dependent pathways. Methionine metabolism has multiple roles in regulating physiological function. Methionine is the initiating amino acid in protein translation, and restriction of this amino acid in the diet has been shown to reduce protein synthesis ${ }^{[23-}$ ${ }^{27]}$. MR has also been shown to increase activity of pathways involved in protein degradation and recycling including the ubiquitin proteasome system and autophagy consistent with enhanced proteostasis ${ }^{[28]}$. Methionine plays a significant role in the generation of hydrogen sulfide via conversion to cysteine through the transsulfuration pathway. Hydrogen sulfide and its generation has been shown to play a central role in the effects of dietary restriction including calorie restriction $(C R)$ and $M R^{[29]}$. Methionine is also used in the generation of S-adenosyl methionine (SAM) which is the primary methyl group donor for methyltransferases. The effects of MR on this metabolite are complex with levels being decreased in some cases ${ }^{[30-32]}$ while having no impact in others ${ }^{[31,33]}$, and with MR either increasing global DNA methylation ${ }^{[30]}$ or having no effect ${ }^{[12,30]}$. There is also evidence that other interventions that extend lifespan significantly alter methionine metabolism. For example, Ames dwarf mice, which live more than $40 \%$ longer than their controls due to a mutation in Prop1, have also been shown to have altered sulfur metabolism and higher SAM turnover ${ }^{[34,35]}$. Interestingly, Ames dwarf mice show no impact of MR on glucose metabolism ${ }^{[36]}$ or longevity ${ }^{[34]}$ suggesting perhaps overlap in pro-longevity mechanisms of these interventions.

The potential role of methionine reduction-oxidation (redox) regulation in the mechanism of MR has been relatively unexplored. Methionine itself is extremely susceptible to oxidation due to the sulfur atom that makes up its amino acid side chain. Under oxidizing conditions, the pro-chiral sulfur of methionine's thioether can be oxidized to yield two epimers of L-methionine sulfoxide ${ }^{[37]}$. Methionine sulfoxide reductases (Msr) have evolved to catalytically reduce methionine sulfoxide to methionine. This is an important oxidative damage repair mechanism as methionine and methionine sulfoxide differ in structure and polarity. In addition, there is growing evidence that redox regulation of methionine residues may also act as functional regulators of proteins $^{[38-42]}$. At least four forms of Msr have evolved in mammals: MsrA which reduces the chiral (S) epimer of methionine sulfoxide and MsrB1-3 which reduce the (R) epimer. Of these, MsrA is the most ubiquitously distributed among mammalian tissues and the most well-studied Msr. The lack of MsrA does increase sensitivity to oxidative challenge and results in increased oxidation of methionine residues ${ }^{[43]}$. Interestingly, MsrA has also been reported to play a role in the regulation of glucose and insulin signaling under metabolic challenge. The lack of MsrA in mice exacerbates insulin resistance caused by high fat feeding in part by failing to prevent oxidative damage to key insulin signaling proteins ${ }^{[44]}$. Conversely, overexpression of MsrA preserves insulin sensitivity in mice fed a high fat diet ${ }^{[45]}$.

Relatively little is known regarding the potential role of methionine redox metabolism or Msr activity in the outcomes of MR. MR in mice reduces plasma methionine and methionine sulfoxide levels to approximately the same degree ${ }^{[31]}$. It has also been shown that the lack of both MsrA and MsrB1, but not either independently, can hinder growth rate of weaned mice under $\mathrm{MR}^{[33]}$, suggesting an important role of Msr enzymes in preserving methionine during restriction. Here, we directly tested the functional requirement for MsrA on the metabolic outcomes of MR in mice. Using mice lacking MsrA expression (MsrA KO), we find that MsrA is not required to reduce body weight or composition under MR. Moreover MsrA KO mice responded as well, or better, to MR in terms of improvement in glucose metabolism and respiration. Together, our studies suggest that functional MsrA is not required for the metabolic benefits of MR.

\section{Results:}

In this study, we used adult mice to potentially differentiate the effects of MR on metabolic function from those of MR-mediated delays in development ${ }^{[2]}$. At an average 7.5 months of age, fully grown mice began 
dietary interventions of either MR $(0.15 \%$ methionine as proportion of protein) or composition equivalent control diet (CD) which was replete with levels of methionine $(0.86 \%$ methionine as proportion of protein) approximately equivalent to that found in normal chow. During the study, diets were provided ad libitum and food consumption was monitored throughout. Prior to intervention with each diet, both male and female MsrA $\mathrm{KO}$ mice were lower in body weight than their wild type counterparts (Fig. 1a, b). In wild type males, MR reduced body weight and fat mass compared to $C D$, consistent with previous reports ${ }^{[1,5,6,17,18,21,22,30]}$. In addition, MR caused a small change in lean mass compared to CD. Surprisingly, MR induced reduction of male body weight, fat mass, and lean mass in MsrA KO to a greater extent than in wild type mice - the percentage change in these outcomes was larger in MsrA KO males compared to wild type males (Fig. 1a, c, e). MR did not result in significant changes body weight, fat mass, or lean mass regardless of genotype during the 3 months of treatment in females (Fig. 1d, f).

Despite the reported body weight reduction in the MR groups, we also found that MR increased food consumption in all MR groups compared to their sex- or genotype-equivalent CD groups (Fig. $1 \mathrm{~g}, \mathrm{~h}$ ). This increase in food consumption when normalized to body weight was stable throughout the three months the mice were on the diet. While MR significantly increased food consumption compared to CD, post-hoc analysis of these data indicated that only the wild type males had a significant difference between the MR and CD. These results are similar to others reported in regards to body composition and food consumption under $\mathrm{MR}^{[1,5,6,17,18,21,22,24,30]}$.

The post-mortem tissue masses reflected the decreased fat mass observed with QMR (Fig. 1e, f) with epididymal and subcutaneous white adipose tissue (WAT) being lower for the MsrA KO males, but with only a small difference observed in the MsrA KO female subcutaneous WAT (Fig. 2a, b). Brown adipose tissue (BAT) was significantly increased in females by MR (Fig. 2c) but not males; previous studies have been equivocal in reporting this the response of BAT to $\mathrm{MR}^{[4,16,46]}$. Surprisingly, MR resulted in a dramatic decrease in brain mass for the male MsrA KO mice, but the reason for this is unclear (Fig. 2d). MR also resulted in sex-specific decreases in liver and kidney mass - a decrease in liver mass in the females and a decrease in kidney mass in the males (Fig. 2e, f). The change in heart mass and gastrocnemius mass (Fig. $2 \mathrm{~g}, \mathrm{~h}$ ) was consistent with the lean mass decrease observed with QMR (Fig. 1c, d).

Similar to previously reports, glucose metabolism was improved by MR even when started in nonobese adult animals ${ }^{[4-6,9,22]}$. In males, glucose tolerance tested (GTT) after 9 weeks of intervention was improved when MR groups were analyzed as a whole (Fig. 3a, b). Post-hoc analysis of the within-sex, two-way ANOVA showed a significant effect of MR in MsrA KO ( $p=0.0047)$ though not in wild type mice $(p=0.1545)$ (Fig. 3b). MR also improved insulin sensitivity in males as measured by insulin tolerance test (ITT) following 11 weeks of dietary intervention (Fig. 3e, f). Similar to GTT results, there was a significant post-hoc effect of MR on MsrA KO mice ( $p=0.0012)$ though not in wild type mice (Fig. 3f). In contrast to results in males, MR had no significant main effect on GTT or ITT in female mice for either wild type or MsrA KO mice (Fig. 3c, d, g, h). We also measured glycated hemoglobin $\mathrm{A} 1 \mathrm{c}(\mathrm{HbA} 1 \mathrm{c})$ as an additional marker of glucose metabolic function, and found $\mathrm{HbA1}$ c significantly lower in male mice on MR compared to CD (Fig. 3i) but there was no effect of diet in females (Fig. 3j). Interestingly, MsrA KO males had lower HbA1c than their wild type male counterparts overall, although there was no difference between MsrA KO and wild type females.

After 12 weeks of MR, plasma was collected after overnight fast to assess endocrinological effects of MR in wild type and MsrA KO mice. MR significantly reduced insulin concentrations in males compared to CD (Fig. 4a). In females, MR reduced insulin concentration in wild type females but not MsrA KO (Fig. 4b). These results are generally in line with the idea that MR improves glucose metabolic function in vivo ${ }^{[4-6,9,22]}$. Leptin, a peptide secreted from adipose tissue and involved in satiety, was lower in the MR males compared to CD males, but unchanged by diet in females (Fig. 4c, d). Serum concentrations of IGF-1 were decreased with MR in both sexes as has been shown in other studies ${ }^{[2,8,46-49]}$, however there was only a significant genotype effect of the MsrA KO in the males (Fig. 4e, f). The effect of MR on other endocrine factors associate with metabolic function were largely unchanged in with diet or genotype in male mice. However, MR did affect these outcomes in female mice with both genotype and diet dependent effects (Supplemental Fig. S1).

We also addressed the effect of MR and lack of MsrA on whole animal metabolism as an additional assessment of metabolic function. Using an independent cohort of mice treated identically, we measured respiration of animals following 8 months of MR. Overall, MR did not affect respiratory exchange ratio (RER) compared to CD (Fig. 5) with the exception of a decrease in dark cycle RER in MsrA KO males on MR (Fig. 5d). Oxygen consumption (VO2) and carbon dioxide production (VCO2) were also analyzed normalized to body weight (Supplemental Fig. S2) and lean mass (Supplemental Fig. S3), with only lean mass normalization showing significant effect of genotype but not diet. Energy expenditure (EE) was calculated from this data and 
averaged over 24 hours, as well as broken into the light and dark cycle averages (Fig. 6). ANCOVA analysis controlling for lean body mass yielded a main effect of the MsrA KO for light cycle EE (Fig. 6e), but had a nearly significant genotype effect for overall $24 \mathrm{hr}$ average EE. Females had a similar outcome with a main effect of MsrA KO for the dark cycle and overall 24hr average EE (Fig. 6d, h). Taken together these results indicate that MR did not significantly alter EE, while MsrA KO had a more profound effect when controlled for lean body mass. (Fig. 6)

\section{Discussion:}

Insight regarding the molecular underpinnings of MR have the potential to significantly advance our understanding of the physiological benefits of this intervention including those on longevity and metabolic function. Here we tested whether the methionine sulfoxide reducing enzyme MsrA impacts the effect of MR on metabolism in mice. Overall, we found that mice lacking MsrA have normal, or potentially even greater, response to MR compared to wild type mice in terms of weight loss, glucose and insulin response, and respiration. One interpretation of these outcomes could be that MsrA is dispensable for the effects of MR on glucose metabolism in vivo, or that the impact of MsrA modulation by MR is on functions that do not directly affect glucose metabolism. In regards to the potential lack of requirement of this enzyme, Zhao et al. showed previously that lack of MsrA did not significantly impact growth from weaning under methionine restricted conditions, a classical bioassay for growth ${ }^{[33]}$. This report showed also that the lack of MsrB1 alone had no effect in this assay, but that the lack of both MsrA and MsrB1 delayed growth under methionine restriction from weaning. Together, these data suggest a functional requirement for Msr in general under conditions of limiting methionine, and also that there may be compensatory mechanisms for this process among Msr enzymes. Data from MsrB1 KO mice showed that other Msr enzymes are upregulated in a tissue specific manner ${ }^{[50]}$. More work in this area would be of benefit to understanding the role of Msr and methionine metabolism in general in MR.

Among potential mechanisms by which MR alters physiological function are reported improvements in resistance and response to oxidative stress induced by $\mathrm{MR}^{[2,10,11,15,43]}$. While not a primary antioxidant, MsrA has been shown to play an important role in the resistance to oxidative stress and the lack of MsrA reduces resistance to oxidant challenge in mice ${ }^{[43,44]}$. Studies have shown that loss of any of these Msr enzymes under normal diet conditions is survivable, but can make the organism more sensitive to oxidative stress.

Counterintuitively, knockout of the four Msr enzymes, MsrA and MsrB1-3, has been shown to increase oxidative stress resistance, presumably through compensatory mechanisms among the antioxidant defense system $^{[51]}$. In addition to repairing oxidized methionine, Msr have been proposed to act more generally in oxidant defense as part of a methionine redox system ${ }^{[52]}$. In this scenario, methionine acts as a "free-radical sink" taking on oxidative damage to protect other macromolecules in the cell. These methionine residues can then be recycled (reduced) by Msr to further defend the cell from oxidant damage. Under this paradigm, it is conceivable that restriction of methionine then increases the importance of functional Msr to prevent oxidative damage. While we did not address this here, it would be of interest to assess the interplay of MR and MsrA under such oxidant challenges.

While our results in general suggest MsrA is not required for the effects of MR on metabolism, some of our results do suggest potential roles in mediating other functions affected by MR. For example, MR in wild type mice did not significantly affect lean mass throughout our study. On the other hand, MsrA KO mice lost a significantly quantity of lean mass under MR that did not return to normal levels through the duration of study (Fig. 1c). Generally, loss of lean mass might be associated with declines in muscle mass, function, etc. We did observe a decrease in gastrocnemius mass at time of tissue collection in both sexes with MR which was significant in MsrA KO post-hoc testing (Fig. 2h). A similar decrease in mass has been reported in other MR studies $^{[4,7,47]}$. Further examination of muscle function and structure will be necessary to delineate this potential novel relationship between methionine metabolism and muscle. In particular, assessment of late-life muscle function and the effect of MR would expand on our understanding of whether this intervention improves overall healthy aging. In addition, it will be of interest to determine whether the MR-mediated declines in kidney, liver, and brain mass of MsrA KO mice have significant impact on the function of these organs in vivo.

In this study, the response to MR on physical and functional outcomes was more pronounced in male mice compared to female mice regardless of genotype. Some evidence of this can be seen in a recent study by Forney et al. which also tested the response to MR in both sexes ${ }^{[17]}$. Similar to our results, this previous study showed that MR has significantly different functional impact on each sex and in general that males respond more robustly to MR than do females. This suggests a significant interaction between these factors 
and the animal's sex, but it remains an open question if this is driven by sex hormones or sex chromosomes. The lack of MsrA had no effect on these sex-differences, suggesting that this enzyme does not affect the central mediators of this MR and genetic sex relationship. Further experiments would be required to better understand the interactions of methionine sulfoxide reductases and sex with the effects of MR.

Overall, our results indicate that 1) MsrA is not functionally required for the effects of MR on metabolic function; 2) MR can significantly improve body composition and metabolic function even when administered to normal adult rodents; 3 ) in general, males respond to a greater degree to MR than do females in terms of metabolic function. While our studies investigated these interactions and effects in adult mice, it remains an open question as to their long-term effects on longevity and health span. While previous studies have indicated that lack of MsrA does not negatively impact lifespan, its interaction with MR is uncertain.

\section{Methods:}

\section{Animal Usage and Ethical Procedures}

All animal experiments were approved by the Institutional Animal Care and Use Committees and UTHSA (Animal Protocol 20170190AR), and have been reported following ARRIVE guidelines. All methods were conducted in accordance with international ethical standards and guidelines.

\section{Animal Care}

Cohort 1:

Genetic mutant mice with homozygous deletion of MsrA were maintained on C57BI/6J background as previously reported ${ }^{[44]}$. For this study, both male and female mice were used, with wild-type C57BI/6J as controls. All mice were confirmed for genotype by PCR analysis of tail-derived genomic DNA. Animal studies were performed in a specific pathogen-free vivarium maintained at $25^{\circ} \mathrm{C}$ with a $12 \mathrm{hr}: 12 \mathrm{hr}$ light:dark cycle. Mice were maintained in ventilator cages at density of 3-4 (male) or 5 (female) mice per cage, and provided food and water ad libitum throughout study except prior to metabolic assessment. Mice were maintained on standard chow (NIA-31 equivalent) until an age of approximately 7.5 months post-weaning until cages were assigned to either control diet (CD) (0.86\% Met, TestDiet 578F w/0.86\% MET - 5SFD) or MR $(0.15 \%$ Met, $0 \%$ Cys, Test Diet 96D2, modified TestDiet 58B0) diet for the duration of the study. 9-10 mice were assigned to each group combination of sex, genotype, and diet, resulting in 8 groups of 9-10 mice. Cages were assigned non-randomly to have approximately equal starting weights between diet groups within each genotype. Body weight and composition (by quantitative magnetic resonance, Echo MRI, Houston TX) were performed prior to initiation of dietary intervention. During the study, mice were weighed weekly, food consumption was measured weekly, and body composition was measured bi-weekly. Tissues were collected after euthanasia via $\mathrm{CO} 2$ asphyxiation, and measured for mass before being snap frozen in liquid nitrogen for storage. During the course of the study one male mouse suffered from rectal prolapse and subsequent weight loss. Data collected from this mouse was censored from the study.

\section{Cohort 2:}

An identical cohort of mice was started at the same time using the same experimental paradigm. These mice were used for measurement of oxygen consumption after being treated with MR or CD for approximately 8 months. Body weight and food consumption were measured in these mice on the same schedule as the first group. Body composition was measured monthly.

Glucose Tolerance (GTT) and Insulin Tolerance Tests (ITT)

Following an overnight (16 hour) fast, fasting blood glucose concentrations were measured by tail bleed using an AimStrip Plus digital glucose meter. For GTT mice injected intraperitoneally with $1.5 \mathrm{mg} / \mathrm{g}$ body weight glucose in PBS. Blood glucose was measured by tail bleed at 15, 30, 60, 90, and 120 minutes post injection. Area Under the Curve (AUC) was calculated via the trapezoid method. ITT were performed similarly with intraperitoneal injection of $0.75 \mathrm{U}$ Insulin/g body weight in PBS and measurement of blood glucose by tail bleed following 15, 30, 60, 90, and 120 minutes. GTT were performed two weeks prior to ITT in the same animals.

\section{Hemoglobin A1C}

At the completion of study, mice were fasted overnight and tail bleed was performed to collect whole blood in EDTA washed tubes and temporarily stored on ice. Collected blood was allowed to warm to room temperature before $\mathrm{HbA} 1 \mathrm{c}$ was measured on a Siemens Vantage DCA Analyzer (Siemens AG, Munich, Germany). 
MilliPlex Metabolic Panel

At the completion of study, mice were fasted overnight. Blood was collected and centrifuged in EDTA-coated tubes then flash frozen. Plasma was then tested in the MilliPlex MMHMAG-44K MILLIPLEX MAP Mouse Metabolic Hormone Panel (EMD Millipore). Data was log transformed to preserve normality prior to analysis.

\section{ELISA}

IGF1 ELISA (Abcam, ab100695) was performed as per manufacturer's instructions.

\section{Oxygen Consumption}

Oxygen consumption was performed on mice after eight months on diet. Oxygen consumption and carbon dioxide generation was measured via the CLAMS system (Columbus Instruments). Mice were individually housed during testing and were housed for $24 \mathrm{hrs}$ before measurement was started. Oxygen and carbon dioxide volume were measured for the next $24 \mathrm{hrs}$ after acclimatization. Measurements were based on $1 \mathrm{hr}$ bins.

\section{Energy Expenditure}

Energy expenditure was calculated based on lean mass from QMR and VO2 following equations provided by the CLAMS system. Briefly, EE was calculated based as $3.815+1.232{ }^{*}$ RER * VO2, with VO2 not normalized to mass.

\section{Statistics}

Statistics were completed using Prism 8. Physiological measures of body weight/composition and food consumption were measured within sex via a Repeated Measures, 3-Way ANOVA with post-hoc multiple comparisons performed with the False Discovery Rate set $Q=0.05$. All other tests were performed using 2Way ANOVA within each sex with post hoc tests to assess diet effect, Sidac corrected. Energy Expenditure was analyzed with the "car" package in R to perform an ANCOVA controlling for lean body weight as a covariate.

\section{References}

1. John P. Richie Jr. et al. Methionine restriction increases blood glutathione and longevity in F344 rats.

FASEB 8, 1302-1307 (1994).

2. Richard A. Miller et al. Methionine-deficient diet extends mouse lifespan, slows immune and lens aging, alters glucose, T4, IGF-I and insulin levels, and increases hepatocyte MIF levels and stress resistance. Aging Cell 4, 119-125 (2005).

3. Sun, L., Sadighi Akha, A. A., Miller, R. A. \& Harper, J. M. Life-Span Extension in Mice by Preweaning Food Restriction and by Methionine Restriction in Middle Age. J. Gerontol. A. Biol. Sci. Med. Sci. 64A, 711-722 (2009).

4. Beatriz A. Guerra et al. Dietary sulfur amino acid restriction upregulates DICER to confer beneficial effects.

Mol. Metab. 29, 124-135 (2019).

5. Louise Grant et al. Methionine restriction improves renal insulin signalling in aged kidneys. Mech. Aging

Dev. 157, 35-43 (2016). 
6. Emma K. Lees et al. Methionine restriction restores a younger metabolic phenotype in adult mice with alterations in fibroblast growth factor 21. Aging Cell 13, 817-827 (2014).

7. Swaminathan, A., Fokin, A., Venckūnas, T. \& Degens, H. Methionine restriction plus overload improves skeletal muscle and metabolic health in old mice on a high fat diet. Sci. Rep. 11, 1260 (2021).

8. Ables, G. P., Perrone, C. E., Orentreich, D. \& Orentreich, N. Methionine-Restricted C57BL/6J Mice Are Resistant to Diet-Induced Obesity and Insulin Resistance but Have Low Bone Density. PLoS ONE 7, e51357 (2012).

9. Stone, K. P., Wanders, D., Orgeron, M., Cortez, C. C. \& Gettys, T. W. Mechanisms of Increased In Vivo Insulin Sensitivity by Dietary Methionine Restriction in Mice. Diabetes 63, 3721-3733 (2014).

10. Gomez, A. et al. Cysteine dietary supplementation reverses the decrease in mitochondrial ROS production at complex I induced by methionine restriction. J. Bioenerg. Biomembr. 47, 199-208 (2015).

11. Ying, Y. et al. Dietary l-methionine restriction decreases oxidative stress in porcine liver mitochondria. Exp. Gerontol. 65, 35-41 (2015).

12. Sanchez-Roman, I. et al. Effects of aging and methionine restriction applied at old age on ROS generation and oxidative damage in rat liver mitochondria. Biogerontology 13, 399-411 (2012).

13. Caro, P. et al. Forty percent and eighty percent methionine restriction decrease mitochondrial ROS generation and oxidative stress in rat liver. Biogerontology 9, 183-196 (2008).

14. Sanchez-Roman, I. et al. Forty percent methionine restriction lowers DNA methylation, complex I ROS generation, and oxidative damage to mtDNA and mitochondrial proteins in rat heart. J. Bioenerg. Biomembr. 43, 699-708 (2011).

15. Sanz, A. et al. Methionine restriction decreases mitochondrial oxygen radical generation and leak as well as oxidative damage to mitochondrial DNA and proteins. FASEB J. 20, 1064-1073 (2006).

16. Yuvraj N. Patil, Kelly N. Dille, David H. Burk, Cory C. Cortez, \& Thomas W. Gettys. Cellular and molecular remodeling of inguinal adipose tissue mitochondria by dietary methionine restriction. J. Nutr. Biochem. 26, 1235-1247 (2015).

17. Forney, L. A. et al. Sexually Dimorphic Effects of Dietary Methionine Restriction are Dependent on Age when the Diet is Introduced. Obesity 28, 581-589 (2020). 
18. Barbara E. Hasek et al. Dietary methionine restriction enhances metabolic flexibility and increases uncoupled respiration in both fed and fasted states. Am J Physiol Regul Integr Comp Physiol 299, R728R739 (2010).

19. Desiree Wanders et al. FGF21 Mediates the Thermogenic and Insulin-Sensitizing Effects of Dietary Methionine Restriction but Not Its Effects on Hepatic Lipid Metabolism. Diabetes 66, 858-867 (2017).

20. Wanders, D. et al. Role of GCN2-Independent Signaling Through a Noncanonical PERK/NRF2 Pathway in the Physiological Responses to Dietary Methionine Restriction. Diabetes 65, 1499-1510 (2016).

21. Plaisance, E. P. et al. Role of $\beta$-adrenergic receptors in the hyperphagic and hypermetabolic responses to dietary methionine restriction. Am. J. Physiol.-Regul. Integr. Comp. Physiol. 299, R740-R750 (2010).

22. Forney, L. A., Wanders, D., Stone, K. P., Pierse, A. \& Gettys, T. W. Concentration-dependent linkage of dietary methionine restriction to the components of its metabolic phenotype: Concentration Dependence of Methionine Restriction. Obesity 25, 730-738 (2017).

23. Rafał Kozieł et al. Methionine restriction slows down senescence in human diploid fibroblasts. Aging Cell 13, 1038-1048 (2014).

24. Pettit, A. P. et al. Dietary Methionine Restriction Regulates Liver Protein Synthesis and Gene Expression Independently of Eukaryotic Initiation Factor 2 Phosphorylation in Mice. J. Nutr. 147, 1031-1040 (2017).

25. Sikalidis, A. K., Mazor, K. M., Kang, M., Liu, H. \& Stipanuk, M. H. Total 4EBP1 Is Elevated in Liver of Rats in Response to Low Sulfur Amino Acid Intake. J. Amino Acids 2013, 1-11 (2013).

26. Nichenametla, S. N., Mattocks, D. A. L., Malloy, V. L. \& Pinto, J. T. Sulfur amino acid restriction-induced changes in redox-sensitive proteins are associated with slow protein synthesis rates: Glutathione redox status and protein synthesis rates. Ann. N. Y. Acad. Sci. 1418, 80-94 (2018).

27. Jonsson, W. O. et al. Physiologic Responses to Dietary Sulfur Amino Acid Restriction in Mice Are Influenced by Atf4 Status and Biological Sex. J. Nutr. 151, 785-799 (2021).

28. Ruckenstuhl, C. et al. Lifespan Extension by Methionine Restriction Requires Autophagy-Dependent Vacuolar Acidification. PLoS Genet. 10, e1004347 (2014).

29. Christopher Hine et al. Endogenous Hydrogen Sulfide Production Is Essential for Dietary Restriction Benefits. Cell 160, 132-144 (2015). 
30. Dwight A.L. Mattocks et al. Short term methionine restriction increases hepatic global DNA methylation in adult but not young male C57BL/6J mice. Exp. Gerontol. 88, 1-8 (2016).

31. Gao, X. et al. Dietary methionine influences therapy in mouse cancer models and alters human metabolism. Nature 572, 397-401 (2019).

32. Tamanna, N., Mayengbam, S., House, J. D. \& Treberg, J. R. Methionine restriction leads to hyperhomocysteinemia and alters hepatic H2S production capacity in Fischer-344 rats. Mech. Ageing Dev. 176, 9-18 (2018).

33. Zhao, H., Kim, G. \& Levine, R. L. Methionine sulfoxide reductase contributes to meeting dietary methionine requirements. Arch. Biochem. Biophys. 522, 37-43 (2012).

34. Brown-Borg, H. M. et al. Growth hormone signaling is necessary for lifespan extension by dietary methionine. Aging Cell 13, 1019-1027 (2014).

35. Eric O. Uthus \& Holly M. Brown-Borg. Methionine flux to transsulfuration is enhanced in the long living Ames dwarf mouse. Mech. Aging Dev. 127, 444-450 (2006).

36. Brown-Borg, H. M., Rakoczy, S., Wonderlich, J. A., Armstrong, V. \& Rojanathammanee, L. Altered dietary methionine differentially impacts glutathione and methionine metabolism in long-living growth hormonedeficient Ames dwarf and wild-type mice. Longev. Heal. 3, 10 (2014).

37. Ronald Bentley. Methionine and Derivatives: Exploring Chirality at Sulfur. Biochem. Mol. Biol. Educ. 33, 274-276 (2005).

38. Ciorba, M. A., Heinemann, S. H., Weissbach, H., Brot, N. \& Hoshi, T. Modulation of potassium channel function by methionine oxidation and reduction. Proc. Natl. Acad. Sci. 94, 9932-9937 (1997).

39. Gao, J. et al. Loss of Conformational Stability in Calmodulin upon Methionine Oxidation. Biophys. J. 74, 1115-1134 (1998).

40. Ciorba, M. A., Heinemann, S. H., Weissbach, H., Brot, N. \& Hoshi, T. Regulation of voltage-dependent K+ channels by methionine oxidation: effect of nitric oxide and vitamin C. FEBS Lett. 442, 48-52 (1999).

41. Dow, L. K., Changela, A., Hefner, H. E. \& Churchill, M. E. A. Oxidation of a critical methionine modulates DNA binding of the Drosophila melanogaster high mobility group protein, HMG-D. FEBS Lett. 414, 514520 (1997). 
42. Fricke, T. C. et al. Oxidation of methionine residues activates the high-threshold heat-sensitive ion channel TRPV2. Proc. Natl. Acad. Sci. 116, 24359-24365 (2019).

43. Salmon, A. B. et al. Lack of methionine sulfoxide reductase A in mice increases sensitivity to oxidative stress but does not diminish life span. FASEB J. 23, 3601-3608 (2009).

44. Styskal, J. et al. Methionine sulfoxide reductase A affects insulin resistance by protecting insulin receptorfunction. Free Radic. Biol. Med. 56, 123-132 (2013).

45. Hunnicut, J., Liu, Y., Richardson, A. \& Salmon, A. B. MsrA Overexpression Targeted to the Mitochondria, but Not Cytosol, Preserves Insulin Sensitivity in Diet-Induced Obese Mice. PLOS ONE 10, e0139844 (2015).

46. Amany K. Elshorbagy et al. Cysteine supplementation reverses methionine restriction effects on rat adiposity: signifi cance of stearoyl-coenzyme A desaturase. J. Lipid Res. 52, 104-112 (2011).

47. J. R. Hens et al. Methionine-restricted diet inhibits growth of MCF10AT1-derived mammary tumors by increasing cell cycle inhibitors in athymic nude mice. BMC Cancer 16, (2016).

48. Malloy, V. L. et al. Methionine restriction decreases visceral fat mass and preserves insulin action in aging male Fischer 344 rats independent of energy restriction. Aging Cell 5, 305-314 (2006).

49. Nichenametla, S. N., Mattocks, D. A. L. \& Malloy, V. L. Age-at-onset-dependent effects of sulfur amino acid restriction on markers of growth and stress in male F344 rats. Aging Cell 19, (2020).

50. Fomenko, D. E. et al. MsrB1 (Methionine-R-sulfoxide Reductase 1) Knock-out Mice: ROLES OF MsrB1 IN REDOX REGULATION AND IDENTIFICATION OF A NOVEL SELENOPROTEIN FORM. J. Biol. Chem. 284, 5986-5993 (2009).

51. Lai, L. et al. Loss of methionine sulfoxide reductases increases resistance to oxidative stress. Free Radic. Biol. Med. 145, 374-384 (2019).

52. R. Abhilash Kumar, Ahmet Koc, Ronald L. Cerny, \& Vadim N. Gladyshev. Reaction Mechanism, Evolutionary Analysis, and Role of Zinc in Drosophila Methionine-R-sulfoxide Reductase. J. Biol. Chem. 277, 37527-37535 (2002).

\section{Acknowledgements}

We would also like to acknowledge the experimental assistance of Yuhong Liu and Jodie Cropper throughout this study, and Dr. Jonathan Dorigatti for experimental assistance and helpful discussions. Milliplex analyte 
panels were performed by Bioanalytics and Single-Cell (BASiC) Core at UT-Health San Antonio. Metabolic cage assessments were performed by the Integrated Physiology of Aging Core at the San Antonio Nathan Shock Center (P30 AG013319). This research was funded in part by R01 AG050797, R01 AG057431, T32 AG021890 and the San Antonio Area Foundation. ABS is partially supported by the Geriatric Research, Education and Clinical Center of the South Texas Veterans Health Care System. This material is the result of work supported with resources and the use of facilities at South Texas Veterans Health Care System, San Antonio, Texas. The contents do not represent the views of the U.S. Department of Veterans Affairs or the United States Government.

\section{Author Contributions}

Study was formulated by ABS and KMT. Data was collected and analyzed by KMT. Figures and manuscript drafts were prepared by KMT. ABS and KMT reviewed, edited, and approved the manuscript.

\section{Competing Interests}

The authors declare no competing interests.

\section{Data Availability}

The data presented in the work are available from the corresponding author upon request.

\section{Figure Legends:}

Figure 1 - MR has sex-specific effects on physiological measures Body weight (a, b) and normalized food consumption $(\mathbf{g}, \mathbf{h})$ measured weekly while on MR diet. Body composition data for fat mass $(\mathbf{c}, \mathbf{d})$, lean mass $(\mathbf{e}, \mathbf{f})$ measured every two weeks. Sexes analyzed separately with Repeated Measures, 3-Way ANOVA. Main effects for diet and MsrA KO genotype indicated by significance bars by legends (right side and above, respectively). Post-hoc diet specific effect within a genotype was analyzed with Repeated Measures, 2-Way ANOVA Multiple Comparisons with False Discovery Rate correction, $Q=0.05$. Means at specific time points being significantly different $(p<0.05)$ between diets for wild type denoted by dollar sign (\$) and for MsrA KO denoted by a pound sign (\#). Graphs represent means \pm s.e.m, and all groups were $8-10$ mice. $\left({ }^{*} p<0.05 ;{ }^{* *} p<0.01 ;{ }^{* * *} p<0.001 ;{ }^{* * * *} p<0.0001\right)$

\section{Figure 2 - MR has sex-specific effects on tissue masses}

Tissue masses for Epididymal WAT (a), Subcutaneous WAT (b), BAT (c), Brain (d), Liver (e), Kidney (f), Heart $(\mathbf{g})$, and Gastrocnemius (h) were measured at time of collection. Analysis was within each sex via Two-Way ANOVA for main effects. Post-hoc analysis was performed with Sidac multiple comparisons correction to assess the diet effect within each genotype. Graphs represent means \pm s.d. and all groups were 8-10 mice. ( ${ }^{*} p$ $<0.05 ;{ }^{* *} p<0.01 ;{ }^{* * *} p<0.001 ;{ }^{* * * *} p<0.0001$ )

\section{Figure 3 - Males have greater glucose metabolism response to MR than females}

Glucose Tolerance Test curves (a, c) and Area Under the Curve (AUC) for their respective curves (b, d) for both sexes. Insulin Tolerance Test curves $(\mathbf{e}, \mathbf{g})$ and AUC for their respective curves (f, $\mathbf{h})$. AUC calculated by the trapezoid method and analyzed within each sex via Two-Way ANOVA for main effects. Post-hoc analysis was performed with Sidac multiple comparisons correction to assess the diet effect within each genotype. $\mathrm{HbA1}$ c was analyzed similarly (i, j). Line graphs represent means \pm s.e.m., bar graphs represent means \pm s.d.. Groups were 8-10 mice. $\left({ }^{*} p<0.05 ;{ }^{* *} p<0.01 ;{ }^{* *} p<0.001\right)$

\section{Figure 4 - MR alters metabolic markers more in males}

MilliPlex of serum from mice overnight fasted at time of sacrifice. Selected panel results of Insulin (a, b) and Leptin (c, d) measured by MilliPlex. Data was log transformed before analysis to preserve normality. Data points at lower end of detection were included as lowest value given by the assay's internal standard curve. IGF-1 (e, f) measured by ELISA. Analysis was within each sex via Two-Way ANOVA for main effects. Post-hoc analysis was performed with Sidac multiple comparisons correction to assess the diet effect within each genotype. Graphs represent means \pm s.d. and all groups were 8-10 mice for MilliPlex, and 5 mice per group for IGF-1 ELISA. $\left({ }^{*} p<0.05 ;{ }^{* *} p<0.01 ;{ }^{* * *} p<0.001\right)$ 
Figure 5 - MR had minimal impact on RER

Respiratory Exchange Ratio (RER) for $24 \mathrm{hr}$ period (a, b), analyzed via 3-Way ANOVA for light and dark cycle independently. Graphs represent mean \pm s.e.m. Male light cycle (c) and dark cycle (d) AUC, and Female light cycle (e) and dark cycle (f) analyzed within each sex via Two-Way ANOVA for main effects on Area Under the Curve (AUC) for RER. Post-hoc analysis was performed with Sidac multiple comparisons correction to assess the diet effect within each genotype. Line graphs represent means \pm s.e.m., bar graphs represent means \pm s.d., and all groups were 9-10 mice on diet for $\sim 8$ months. ( $\left.{ }^{*} p<0.05\right)$

\section{Figure 6 - Energy Expenditure was not impacted by MR}

Energy Expenditure (EE) for 24hr period based on VO2 not normalized to mass for mice from Cohort 2 (a,b). Data averaged over the $24 \mathrm{hr}$ test period (c,d), and for the light $(\mathbf{e}, \mathbf{f})$ and dark $(\mathbf{g}, \mathbf{h})$ cycle separately. Analyzed with ANCOVA using lean mass as a control variable for AUC graphs of EE for full $24 \mathrm{hr}$ as well as light and dark cycles. Line graph represents mean \pm s.e.m., bar graphs represent mean \pm s.d. Groups were 9-10 mice. $\left({ }^{*} p<0.05\right)$ 
Figures

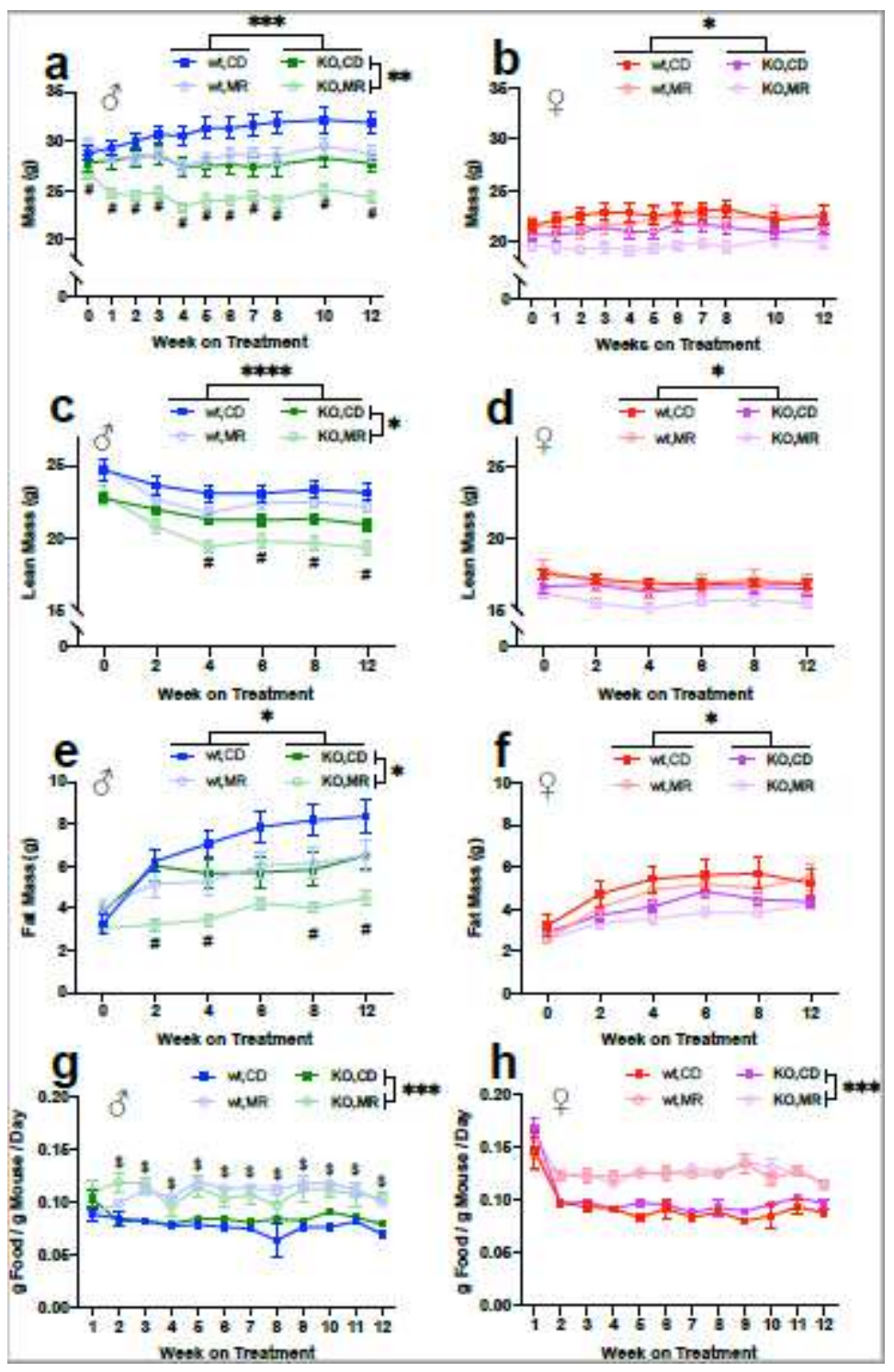

Figure 1

MR has sex-specific effects on physiological measures Body weight $(a, b)$ and normalized food consumption $(\mathrm{g}, \mathrm{h})$ measured weekly while on MR diet. Body composition data for fat mass $(\mathrm{c}, \mathrm{d})$, lean mass (e, f) measured every two weeks. Sexes analyzed separately with Repeated Measures, 3-Way ANOVA. Main effects for diet and MsrA KO genotype indicated by significance bars by legends (right side and above, respectively). Post-hoc diet specific effect within a genotype was analyzed with Repeated Measures, 2-Way ANOVA Multiple Comparisons with False Discovery Rate correction, $Q=0.05$. Means at specific time points being significantly different $(p<0.05)$ between diets for wild type denoted by dollar sign (\$) and for MsrA KO denoted by a pound sign (\#). Graphs represent means \pm s.e.m, and all groups

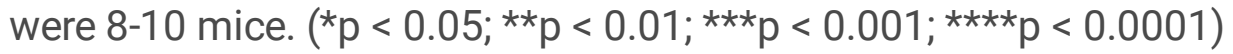




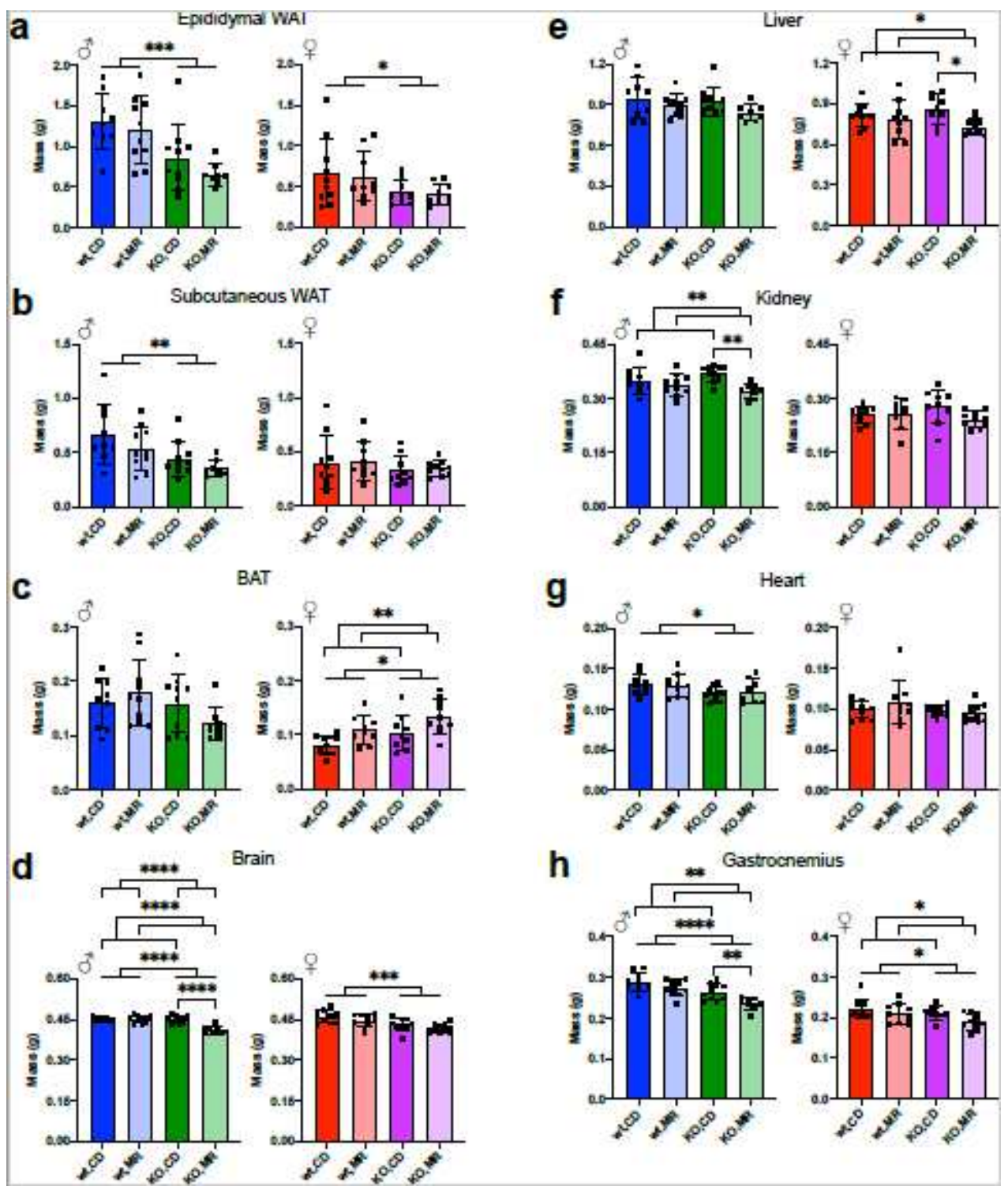

Figure 2

MR has sex-specific effects on tissue masses Tissue masses for Epididymal WAT (a), Subcutaneous WAT (b), BAT (c), Brain (d), Liver (e), Kidney (f), Heart (g), and Gastrocnemius (h) were measured at time of collection. Analysis was within each sex via Two-Way ANOVA for main effects. Post-hoc analysis was performed with Sidac multiple comparisons correction to assess the diet effect within each genotype. Graphs represent means \pm s.d. and all groups were 8-10 mice. $\left({ }^{\star} p<0.05 ; * \star p<0.01 ; * \star \star p<0.001 ; * \star \star \star p<\right.$ 0.0001) 

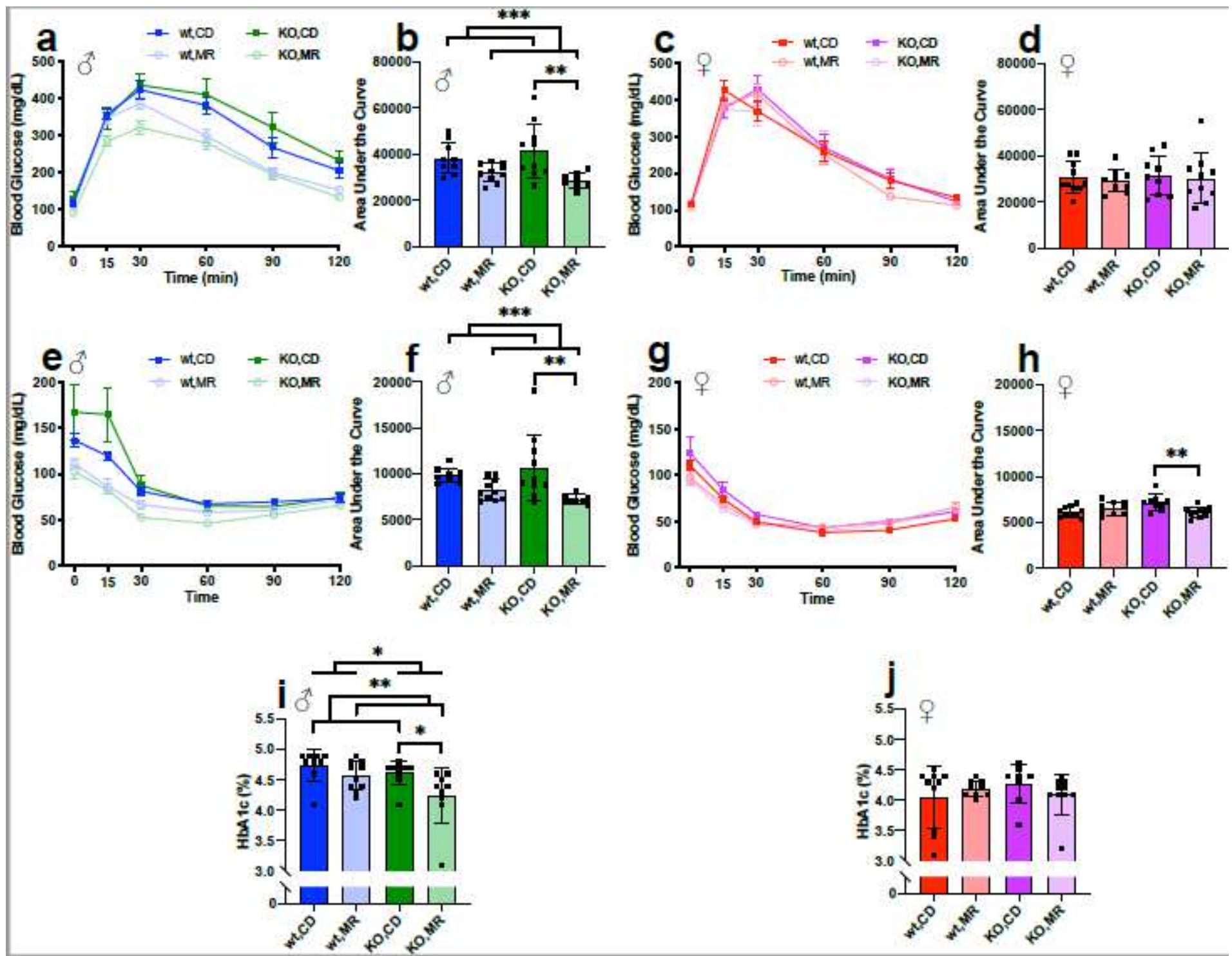

Figure 3

Males have greater glucose metabolism response to MR than females Glucose Tolerance Test curves (a, c) and Area Under the Curve (AUC) for their respective curves (b, d) for both sexes. Insulin Tolerance Test curves $(e, g)$ and AUC for their respective curves $(f, h)$. AUC calculated by the trapezoid method and analyzed within each sex via Two-Way ANOVA for main effects. Post-hoc analysis was performed with Sidac multiple comparisons correction to assess the diet effect within each genotype. HbA1c was analyzed similarly $(i, j)$. Line graphs represent means \pm s.e.m., bar graphs represent means \pm s.d.. Groups were 8-10 mice. $\left({ }^{\star} p<0.05 ;{ }^{* \star} p<0.01 ;{ }^{\star \star \star} p<0.001\right)$ 


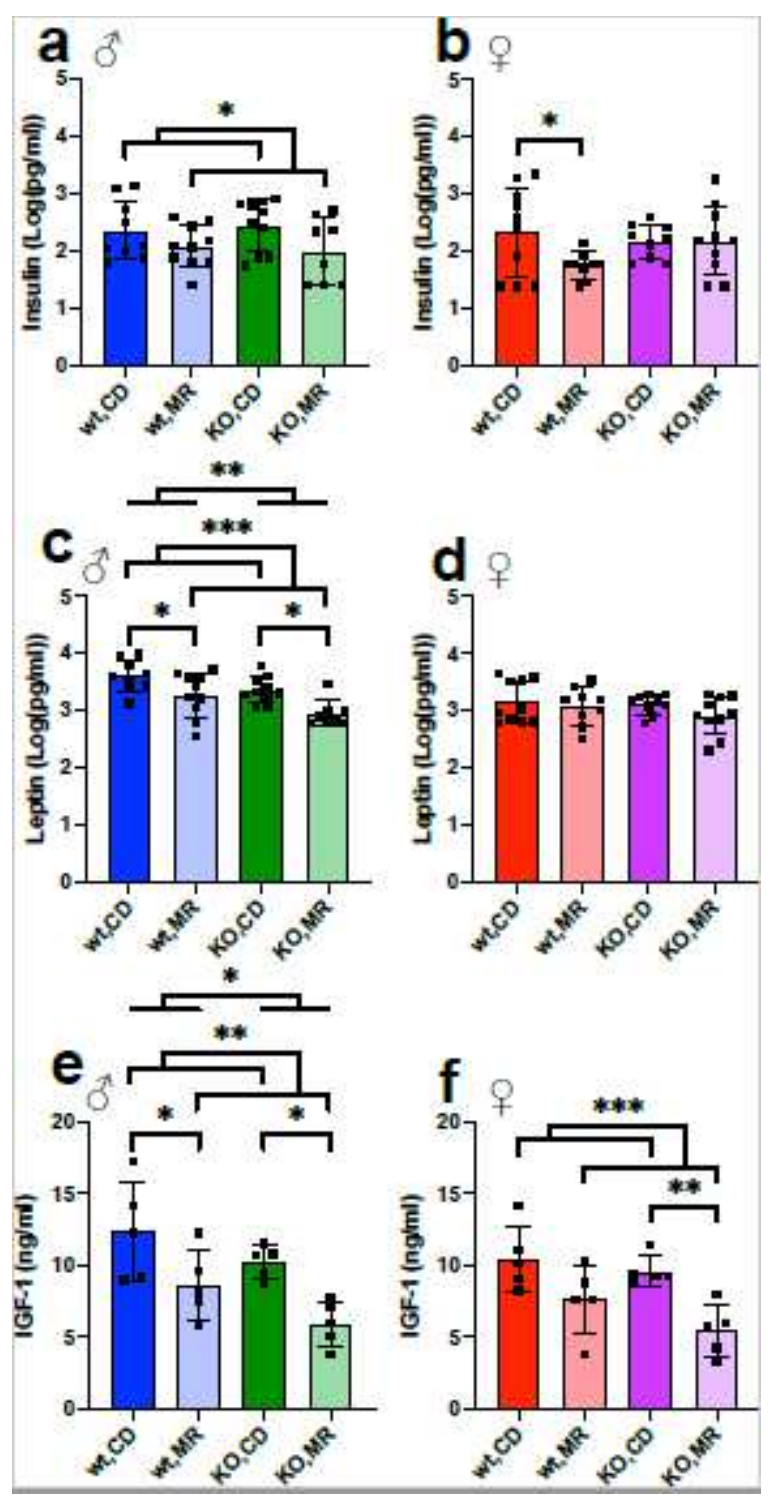

Figure 4

MR alters metabolic markers more in males MilliPlex of serum from mice overnight fasted at time of sacrifice. Selected panel results of Insulin $(a, b)$ and Leptin $(c, d)$ measured by MilliPlex. Data was log transformed before analysis to preserve normality. Data points at lower end of detection were included as lowest value given by the assay's internal standard curve. IGF-1 $(e, f)$ measured by ELISA. Analysis was within each sex via Two-Way ANOVA for main effects. Post-hoc analysis was performed with Sidac multiple comparisons correction to assess the diet effect within each genotype. Graphs represent means \pm s.d. and all groups were 8-10 mice for MilliPlex, and 5 mice per group for IGF-1 ELISA. $\left({ }^{\star} p<0.05 ;{ }^{* *} p<\right.$ $0.01 ; * \star * p<0.001)$ 


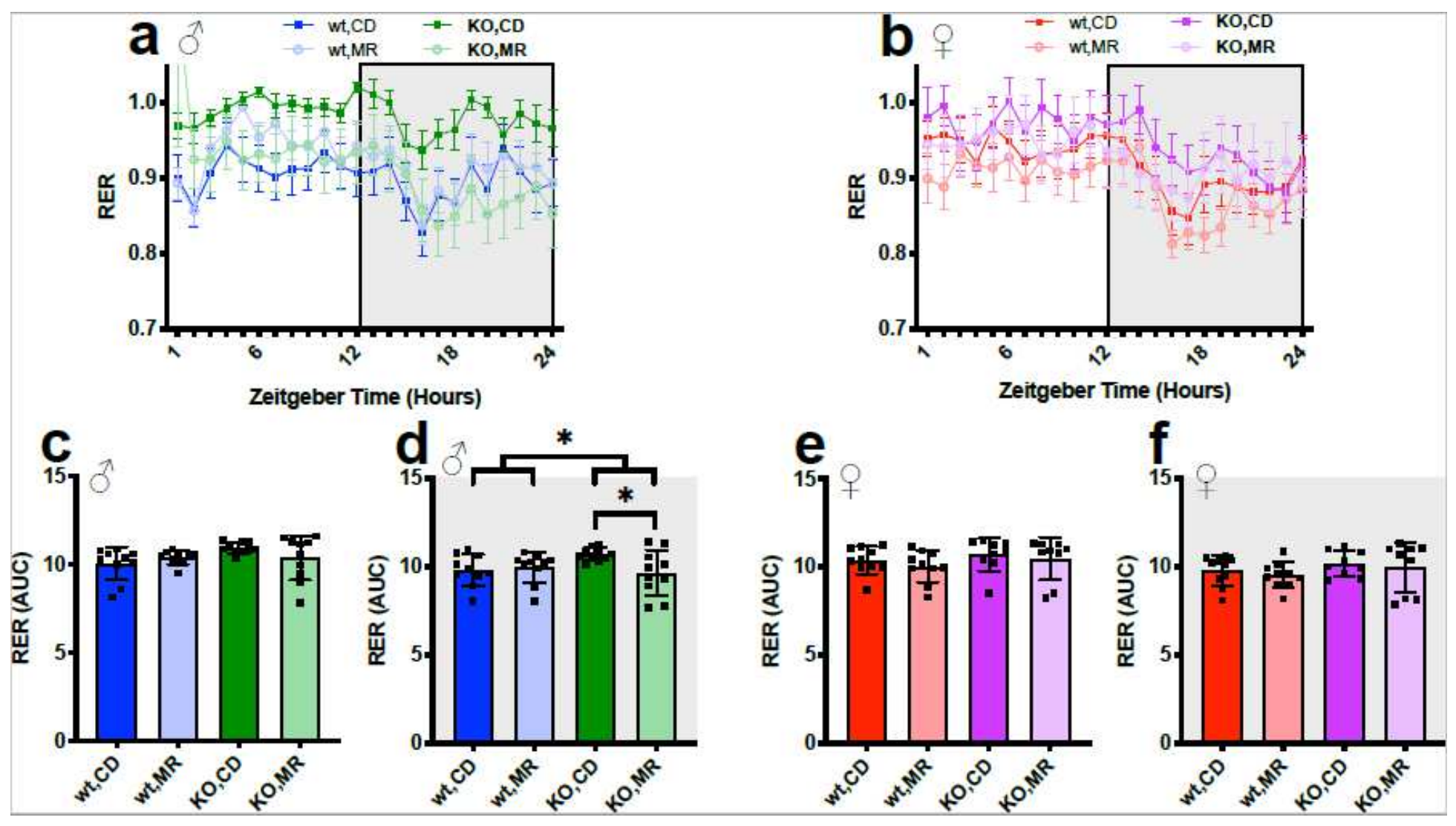

Figure 5

MR had minimal impact on RER Respiratory Exchange Ratio (RER) for 24hr period (a, b), analyzed via 3Way ANOVA for light and dark cycle independently. Graphs represent mean \pm s.e.m. Male light cycle (c) and dark cycle (d) AUC, and Female light cycle (e) and dark cycle (f) analyzed within each sex via TwoWay ANOVA for main effects on Area Under the Curve (AUC) for RER. Post-hoc analysis was performed with Sidac multiple comparisons correction to assess the diet effect within each genotype. Line graphs represent means \pm s.e.m., bar graphs represent means \pm s.d., and all groups were 9-10 mice on diet for $\sim 8$ months. $\left({ }^{\star} \mathrm{p}<0.05\right)$ 

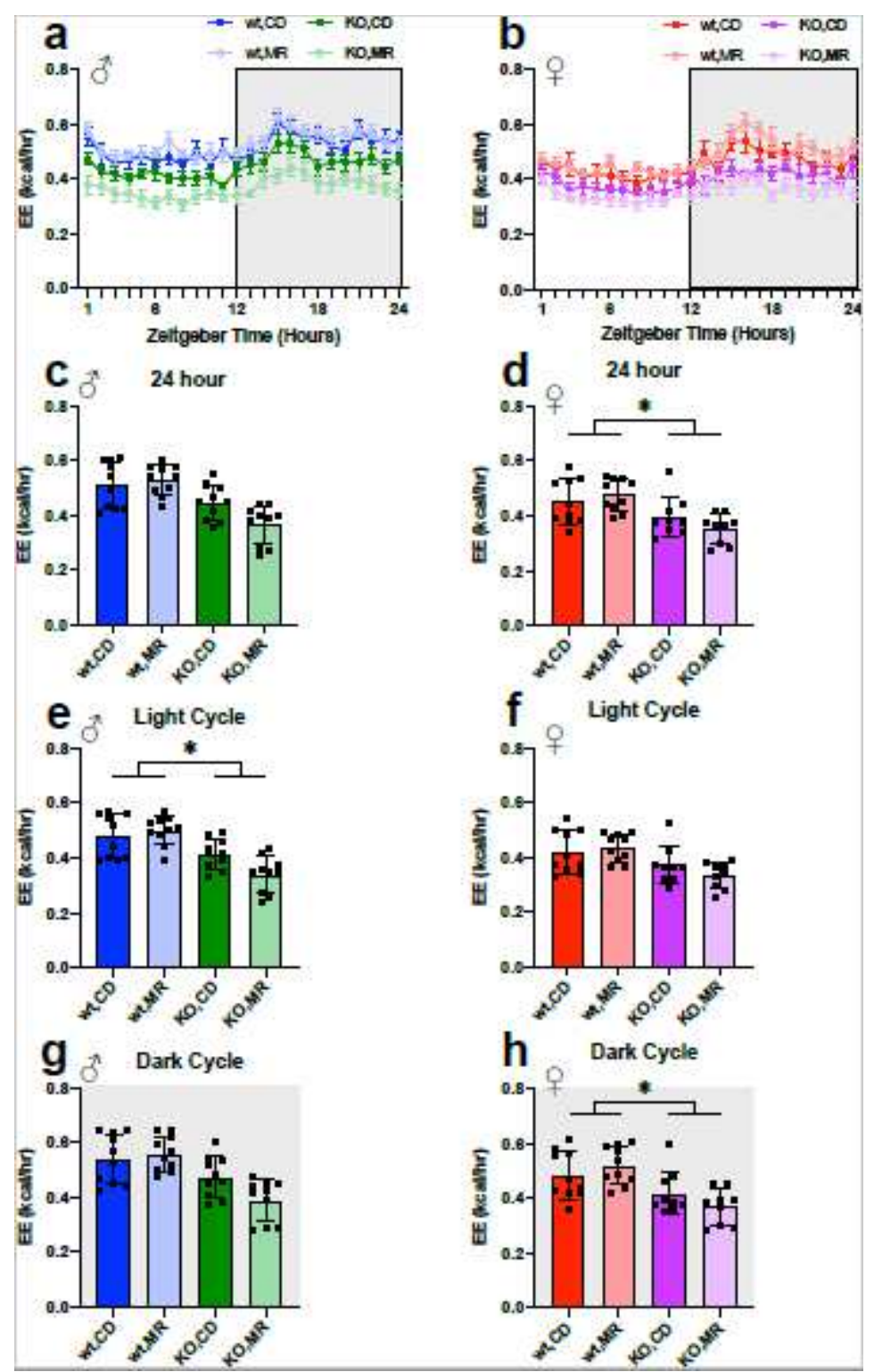

Figure 6

Energy Expenditure was not impacted by MR Energy Expenditure (EE) for $24 \mathrm{hr}$ period based on VO2 not normalized to mass for mice from Cohort $2(a, b)$. Data averaged over the $24 \mathrm{hr}$ test period $(c, d)$, and for the light $(e, f)$ and dark $(g, h)$ cycle separately. Analyzed with ANCOVA using lean mass as a control variable for AUC graphs of EE for full $24 \mathrm{hr}$ as well as light and dark cycles. Line graph represents mean \pm s.e.m., bar graphs represent mean \pm s.d. Groups were $9-10$ mice. $\left({ }^{*} p<0.05\right)$

\section{Supplementary Files}

This is a list of supplementary files associated with this preprint. Click to download.

- SupplementalFigures.pdf 